\title{
Duplicate Form of the Generalized Carlitz Inversions and Summation Formulae
}

\author{
Qiaoying Dong \\ College of Science, Tianjin University of Technology, Tianjin, China \\ Email: qiaoyingdong1123@163.com
}

How to cite this paper: Dong, Q.Y. (2019) Duplicate Form of the Generalized Carlitz Inversions and Summation Formulae. Journal of Applied Mathematics and Physics, 7, 900-911.

https://doi.org/10.4236/jamp.2019.74060

Received: March 25, 2019

Accepted: April 21, 2019

Published: April 24, 2019

Copyright $\odot 2019$ by author(s) and Scientific Research Publishing Inc. This work is licensed under the Creative Commons Attribution International License (CC BY 4.0).

http://creativecommons.org/licenses/by/4.0/

\section{c) (i) Open Access}

\begin{abstract}
The duplicate form of the generalized Gould-Hsu inversions has been obtained by Shi and Zhang. In this paper, we present a simple proof of this duplicate form. With the same method, we construct the duplicate form of the generalized Carlitz inversions. Using this duplicate form, we obtain several terminating basic hypergeometric identities and some limiting cases.
\end{abstract}

\section{Keywords}

Duplicate Inversions, Generalized Gould-Hsu Inversions,

Generalized Carlitz Inversions

\section{Notation and Introduction}

All the notation and terminology is adopted from [1]. Given a complex number $q$ with $|q|<1$, the $q$-shifted factorial is defined for any complex parameter $a$ by

$$
(a ; q)_{0}=1, \quad(a ; q)_{n}=(1-a)(1-a q) \cdots\left(1-a q^{n-1}\right) ;
$$

For notational brevity, we write

$$
(a, b, \cdots, c ; q)_{n}=(a ; q)_{n}(b ; q)_{n} \cdots(c ; q)_{n},
$$

and

$$
\left[\begin{array}{l}
a, b, \cdots, c \\
u, v, \cdots, w
\end{array} ;\right]_{n}=\frac{(a ; q)_{n}(b ; q)_{n} \cdots(c ; q)_{n}}{(u ; q)_{n}(v ; q)_{n} \cdots(w ; q)_{n}} .
$$

The basic hypergeometric series is defined by

$$
\left.{ }_{r} \phi_{s}\left[\begin{array}{l}
a_{1}, \cdots, a_{r} \\
b_{1}, \cdots, b_{s}
\end{array} ;, z\right]=\sum_{k=0}^{\infty} \frac{\left(a_{1}, \cdots, a_{r} ; q\right)_{k}}{\left(b_{1}, \cdots, b_{s} ; q\right)_{k}} \frac{z^{k}}{(q ; q)_{k}}\left((-1)^{k} q^{(k} \begin{array}{l}
k \\
2
\end{array}\right)\right)^{s-r+1} .
$$

Furthermore, Gaussian binomial coefficient is defined by 


$$
\left[\begin{array}{l}
n \\
k
\end{array}\right]= \begin{cases}\frac{(q ; q)_{n}}{(q ; q)_{k}(q ; q)_{n-k}}, & 0<k \leq n ; \\
0, & k<0 \text { or } k>n .\end{cases}
$$

In 1973, Gould and Hsu [2] found their well-known inverse relations. At the same time, Carlitz [3] found the $q$-analogue of Gould-Hsu inversions. Chu [4] extended Gould-Hsu inversions to evaluate the terminating hypergeometric series identities, which can equivalently be reproduced as follows. Let $\left\{a_{k}\right\}_{k \geq 0}$ and $\left\{b_{k}\right\}_{k \geq 0}$ be any two complex sequences such that $\tau$-polynomials defined by

$$
\tau(x ; 0) \equiv 1, \quad \tau(x ; n)=\prod_{k=0}^{n-1}\left(a_{k}+x b_{k}\right), \quad n=1,2, \cdots
$$

where $x$ is an indeterminate and $n$ is a non-negative integer. Let $\lambda$ be a complex number. Then the following inverse relations are equivalent:

$$
\begin{gathered}
F(n)=\sum_{k=0}^{n}(-1)^{k}\left(\begin{array}{l}
n \\
k
\end{array}\right) \tau(\lambda+k ; n) \tau(-k ; n) \frac{\lambda+2 k}{(\lambda+n)_{k+1}} G(k), \\
G(n)=\sum_{k=0}^{n}(-1)^{k}\left(\begin{array}{l}
n \\
k
\end{array}\right) \frac{a_{k}+(\lambda+k) b_{k}}{\tau(\lambda+n ; k+1)} \frac{a_{k}-k b_{k}}{\tau(-n ; k+1)}(\lambda+k)_{n} F(k),
\end{gathered}
$$

provided that the sequence-transforms involved are non-singular; i.e. $\tau(\lambda+n ; m+1), \quad \tau(-n ; m+1), \quad(\lambda+n)_{m+1}$ do not vanish for non-negative integers $m \leq n$, where the shifted-factorial is defined for any complex parameter $a$ by

$$
(a)_{0}=1, \quad(a)_{n}=a(a+1) \cdots(a+n-1) .
$$

Chu [5] also extended Carlitz inverse relations to demonstrate numerous strange $q$-hypergeometric identities, which can be reproduced as follows.

$$
\begin{aligned}
& F(n)=\sum_{k=0}^{n}(-1)^{k}\left[\begin{array}{l}
n \\
k
\end{array}\right] \tau\left(\lambda q^{k} ; n\right) \tau\left(q^{-k} ; n\right) \frac{1-\lambda q^{2 k}}{\left(\lambda q^{n} ; q\right)_{k+1}} G(k), \\
& G(n)=\sum_{k=0}^{n}(-1)^{k}\left[\begin{array}{l}
n \\
k
\end{array}\right] q^{\left(\begin{array}{c}
n-k \\
2
\end{array}\right)} \frac{a_{k}+\lambda q^{k} b_{k}}{\tau\left(\lambda q^{n} ; k+1\right)} \frac{a_{k}+q^{-k} b_{k}}{\tau\left(q^{-n} ; k+1\right)}\left(\lambda q^{k} ; q\right)_{n} F(k),
\end{aligned}
$$

provided that the sequence-transforms involved are non-singular; i.e. $\tau\left(\lambda q^{n} ; m+1\right), \quad \tau\left(q^{-n} ; m+1\right), \quad\left(\lambda q^{n} ; q\right)_{m+1}$ do not vanish for non-negative integers $m \leq n$, where $\tau$-polynomials are defined by (1.1). To evaluate a class of terminating balanced ${ }_{5} F_{4}$-series, the duplicate form of Gould-Hsu inversions has been devised by Chu [6], where the multiplicate form is also constructed. Later, the duplicated form of Carlitz inversions, which has been obtained by Wei et al. [7], is utilized to evaluate three special cases of $q$-Saalschütz theorem. Recently, the triplicate form of Gould-Hsu inversions is used to investigate the dual relations of the Pfaff-Saalschütz summation theorem by Chen and Chu [8] and Wei et al. [9].

In the next section, we use another method to prove the duplicate form of the generalized Gould-Hsu inversions, which have been obtained by Shi and Zhang in [10], and construct the duplicate form of the generalized Carlitz inversions. 
Applying the duplicate inversions to the specialized $q$-Dougall theorem ([5], Equation (4.3a))

$$
\begin{aligned}
& { }_{8} \phi_{7}\left[\begin{array}{c}
a, q a^{\frac{1}{2}},-q a^{\frac{1}{2}}, \frac{a^{2}}{b^{2}}, b q^{\frac{n}{2}}, b q^{\frac{1+n}{2}}, q^{-\frac{n}{2}}, q^{-\frac{n-1}{2}} \\
a^{\frac{1}{2}},-a^{\frac{1}{2}}, \frac{b^{2} q}{a}, \frac{a q^{1-\frac{n}{2}}}{b}, \frac{a q^{\frac{1-n}{2}}}{b}, a q^{1+\frac{n}{2}}, a q^{\frac{1+n}{2}} ; q, q
\end{array}\right] \\
& =\left(-\frac{b}{a}\right)^{n}\left[\begin{array}{c}
a q^{\frac{1}{2}}, \frac{a}{b} \\
\frac{b^{2} q^{\frac{1}{2}}}{a}, \frac{b}{a}
\end{array}\right]_{n}^{\frac{1}{2}}\left[\begin{array}{c}
\frac{b^{2} q^{\frac{1}{2}}}{a} ; q \\
a q^{\frac{1}{2}}
\end{array}\right]_{n},
\end{aligned}
$$

we derive several terminating basic hypergeometric identities in Section 3.

\section{Inverse Relations}

Let $\left\{A_{k}, B_{k}, C_{k}, D_{k}\right\}_{k \geq 0}$ be any four complex sequences such that the $\varphi$-polynomial and $\psi$-polynomial defined by

$$
\begin{gathered}
\varphi(x ; 0) \equiv 1, \quad \varphi(x ; n)=\prod_{k=0}^{n-1}\left(A_{k}+x B_{k}\right), \quad n=1,2, \cdots \\
\psi(x ; 0) \equiv 1, \quad \psi(x ; n)=\prod_{k=0}^{n-1}\left(C_{k}+x D_{k}\right), \quad n=1,2, \cdots
\end{gathered}
$$

where $x$ is an indeterminate and $n$ is a non-negative integer.

Motivated by the proof of Theorem 2.1 in [11], we give a simple proof of duplicate form of generalized Gould-Hsu inversions.

Theorem 2.1. ([10], Theorem 1) (Duplicate form of the generalized Gould-Hsu inversions) With $\varphi$ and $\psi$-polynomials defined respectively by (2.1) and (2.2), the equation

$$
\begin{aligned}
& \Omega(n)=\sum_{k \geq 0}\left(\begin{array}{c}
n \\
2 k
\end{array}\right) \frac{\left(C_{k}+(\lambda+2 k) D_{k}\right)\left(C_{k}-2 k D_{k}\right)(\lambda+2 k)_{n}}{\varphi(-n ; k) \varphi(\lambda+n ; k) \psi(-n ; k+1) \psi(\lambda+n ; k+1)} f(k) \\
& -\sum_{k \geq 0}\left(\begin{array}{c}
n \\
2 k+1
\end{array}\right) \frac{\left(A_{k}+(\lambda+2 k+1) B_{k}\right)\left(A_{k}-(2 k+1) B_{k}\right)(\lambda+2 k+1)_{n}}{\varphi(-n ; k+1) \varphi(\lambda+n ; k+1) \psi(-n ; k+1) \psi(\lambda+n ; k+1)} g(k)
\end{aligned}
$$

is equivalent to the system of equations

$$
\begin{aligned}
f(n)= & \sum_{k=0}^{2 n}(-1)^{k}\left(\begin{array}{c}
2 n \\
k
\end{array}\right) \varphi(-k ; n) \varphi(\lambda+k ; n) \psi(-k ; n) \psi(\lambda+k ; n) \\
& \times \frac{\lambda+2 k}{(\lambda+2 n)_{k+1}} \Omega(k), \\
g(n)= & \sum_{k=0}^{2 n+1}(-1)^{k}\left(\begin{array}{c}
2 n+1 \\
k
\end{array}\right) \varphi(-k ; n) \varphi(\lambda+k ; n) \psi(-k ; n+1) \psi(\lambda+k ; n+1) \\
& \times \frac{\lambda+2 k}{(\lambda+2 n+1)_{k+1}} \Omega(k),
\end{aligned}
$$

provided that the sequence-transforms involved are non-singular, i.e. 
$\varphi(\lambda+n ; m+1), \quad \varphi(-n ; m+1), \quad \varphi(\lambda+n ; m), \quad \varphi(-n ; m), \psi(\lambda+n ; m+1)$, $\psi(-n ; m+1),(\lambda+2 n+1)_{m+1}$ and $(\lambda+2 n)_{m+1}$ do not vanish for non-negative integers $m \leq n$.

Proof. Now substituting (1.1) into (1.2) and (1.3) respectively, we get that

$$
F(n)=\sum_{k=0}^{n}(-1)^{k}\left(\begin{array}{l}
n \\
k
\end{array}\right) \prod_{i=0}^{n-1}\left(a_{i}+(\lambda+k) b_{i}\right)\left(a_{i}-k b_{i}\right) \frac{\lambda+2 k}{(\lambda+n)_{k+1}} G(k)
$$

if and only if

$$
G(n)=\sum_{k=0}^{n}(-1)^{k}\left(\begin{array}{l}
n \\
k
\end{array}\right) \frac{\left(a_{k}+(\lambda+k) b_{k}\right)\left(a_{k}-k b_{k}\right)}{\prod_{i=0}^{k}\left(a_{i}+(\lambda+n) b_{i}\right)\left(a_{i}-n b_{i}\right)}(\lambda+k)_{n} F(k) .
$$

Now we split (2.7) into two sums

$$
\begin{aligned}
G(n)= & \sum_{k \geq 0}\left(\begin{array}{c}
n \\
2 k
\end{array}\right) \frac{\left(a_{2 k}+(\lambda+2 k) b_{2 k}\right)\left(a_{2 k}-2 k b_{2 k}\right)(\lambda+2 k)_{n} F(2 k)}{\prod_{i=0}^{2 k}\left(a_{i}+(\lambda+n) b_{i}\right)\left(a_{i}-n b_{i}\right)} \\
& -\sum_{k \geq 0}\left(\begin{array}{c}
n \\
2 k+1
\end{array}\right) \frac{\left(a_{2 k+1}+(\lambda+2 k+1) b_{2 k+1}\right)\left(a_{2 k+1}-(2 k+1) b_{2 k+1}\right)(\lambda+2 k+1)_{n} F(2 k+1)}{\prod_{i=0}^{2 k+1}\left(a_{i}+(\lambda+n) b_{i}\right)\left(a_{i}-n b_{i}\right)} \\
= & \sum_{k \geq 0}\left(\begin{array}{c}
n \\
2 k
\end{array}\right) \frac{\left(a_{2 k}+(\lambda+2 k) b_{2 k}\right)\left(a_{2 k}-2 k b_{2 k}\right)(\lambda+2 k)_{n} F(2 k)}{\prod_{i=0}^{k-1}\left(a_{2 i+1}+(\lambda+n) b_{2 i+1}\right)\left(a_{2 i+1}-n b_{2 i+1}\right) \prod_{i=0}^{k}\left(a_{2 i}+(\lambda+n) b_{2 i}\right)\left(a_{2 i}-n b_{2 i}\right)} \\
& -\sum_{k \geq 0}\left(\begin{array}{c}
n \\
2 k+1
\end{array}\right) \frac{\left(a_{2 k+1}+(\lambda+2 k+1) b_{2 k+1}\right)\left(a_{2 k+1}-(2 k+1) b_{2 k+1}\right)(\lambda+2 k+1)_{n} F(2 k+1)}{\prod_{i=0}^{k}\left(a_{2 i+1}+(\lambda+n) b_{2 i+1}\right)\left(a_{2 i+1}-n b_{2 i+1}\right)\left(a_{2 i}+(\lambda+n) b_{2 i}\right)\left(a_{2 i}-n b_{2 i}\right)} .
\end{aligned}
$$

Then setting

$$
\begin{aligned}
& a_{2 k}=C_{k}, \quad b_{2 k}=D_{k}, \quad a_{2 k+1}=A_{k}, \quad b_{2 k+1}=B_{k}, \\
& G(n)=\Omega(n), \quad F(2 k)=f(k), \quad F(2 k+1)=g(k)
\end{aligned}
$$

in the last equation, we obtain

$$
\begin{aligned}
& \Omega(n)=\sum_{k \geq 0}\left(\begin{array}{c}
n \\
2 k
\end{array}\right) \frac{\left(C_{k}+(\lambda+2 k) D_{k}\right)\left(C_{k}-2 k D_{k}\right)(\lambda+2 k)_{n}}{\varphi(-n ; k) \varphi(\lambda+n ; k) \psi(-n ; k+1) \psi(\lambda+n ; k+1)} f(k) \\
& -\sum_{k \geq 0}\left(\begin{array}{c}
n \\
2 k+1
\end{array}\right) \frac{\left(A_{k}+(\lambda+2 k+1) B_{k}\right)\left(A_{k}-(2 k+1) B_{k}\right)(\lambda+2 k+1)_{n}}{\varphi(-n ; k+1) \varphi(\lambda+n ; k+1) \psi(-n ; k+1) \psi(\lambda+n ; k+1)} g(k),
\end{aligned}
$$

which is (2.3).

On the other hand, we rewrite (2.6) as

$$
\begin{aligned}
F(2 n)= & \sum_{k=0}^{2 n}(-1)^{k}\left(\begin{array}{c}
2 n \\
k
\end{array}\right) \prod_{i=0}^{2 n-1}\left(a_{i}+(\lambda+k) b_{i}\right)\left(a_{i}-k b_{i}\right) \frac{\lambda+2 k}{(\lambda+2 n)_{k+1}} G(k) \\
= & \sum_{k=0}^{2 n}(-1)^{k}\left(\begin{array}{c}
2 n \\
k
\end{array}\right) \prod_{i=0}^{n-1}\left(a_{2 i+1}+(\lambda+k) b_{2 i+1}\right)\left(a_{2 i+1}-k b_{2 i+1}\right) \\
& \times\left(a_{2 i}+(\lambda+k) b_{2 i}\right)\left(a_{2 i}-k b_{2 i}\right) \frac{\lambda+2 k}{(\lambda+2 n)_{k+1}} G(k),
\end{aligned}
$$

and 


$$
\begin{aligned}
F(2 n+1)= & \sum_{k=0}^{2 n+1}(-1)^{k}\left(\begin{array}{c}
2 n+1 \\
k
\end{array}\right) \prod_{i=0}^{2 n}\left(a_{i}+(\lambda+k) b_{i}\right)\left(a_{i}-k b_{i}\right) \frac{\lambda+2 k}{(\lambda+2 n+1)_{k+1}} G(k) \\
= & \sum_{k=0}^{2 n+1}(-1)^{k}\left(\begin{array}{c}
2 n+1 \\
k
\end{array}\right) \prod_{i=0}^{n-1}\left(a_{2 i+1}+(\lambda+k) b_{2 i+1}\right)\left(a_{2 i+1}-k b_{2 i+1}\right) \\
& \times \prod_{i=0}^{n}\left(a_{2 i}+(\lambda+k) b_{2 i}\right)\left(a_{2 i}-k b_{2 i}\right) \frac{\lambda+2 k}{(\lambda+2 n+1)_{k+1}} G(k) .
\end{aligned}
$$

Using (2.8), we can easily derive (2.4) and (2.5).

Starting with $G(n)$ in (1.4) and carrying out the analogous calculations as in Theorem 2.1, we get the following theorem.

Theorem 2.2. (Duplicate form of the generalized Carlitz inversions) With $\varphi$ and $\psi$-polynomials defined respectively by (2.1) and (2.2), the equation

$$
\begin{aligned}
& \Omega(n)=\sum_{k \geq 0}\left[\begin{array}{c}
n \\
2 k
\end{array}\right] q^{\left(\begin{array}{c}
n-2 k \\
2
\end{array}\right)} \frac{\left(C_{k}+\lambda q^{2 k} D_{k}\right)\left(C_{k}+q^{-2 k} D_{k}\right)\left(\lambda q^{2 k} ; q\right)_{n}}{\varphi\left(q^{-n} ; k\right) \varphi\left(\lambda q^{n} ; k\right) \psi\left(q^{-n} ; k+1\right) \psi\left(\lambda q^{n} ; k+1\right)} f(k) \\
& -\sum_{k \geq 0}\left[\begin{array}{c}
n \\
2 k+1
\end{array}\right] q^{\left(\begin{array}{c}
n-2 k-1 \\
2
\end{array}\right)} \frac{\left(A_{k}+\lambda q^{2 k+1} B_{k}\right)\left(A_{k}+q^{-2 k-1} B_{k}\right)\left(\lambda q^{2 k+1} ; q\right)_{n}}{\varphi\left(q^{-n} ; k+1\right) \varphi\left(\lambda q^{n} ; k+1\right) \psi\left(q^{-n} ; k+1\right) \psi\left(\lambda q^{n} ; k+1\right)} g(k)
\end{aligned}
$$

is equivalent to the system of equations

$$
\begin{aligned}
& f(n)=\sum_{k=0}^{2 n}(-1)^{k}\left[\begin{array}{c}
2 n \\
k
\end{array}\right] \varphi\left(q^{-k} ; n\right) \varphi\left(\lambda q^{k} ; n\right) \psi\left(q^{-k} ; n\right) \psi\left(\lambda q^{k} ; n\right) \times \frac{1-\lambda q^{2 k}}{\left(\lambda q^{2 n} ; q\right)_{k+1}} \Omega(k), \\
& g(n)= \sum_{k=0}^{2 n+1}(-1)^{k}\left[\begin{array}{c}
2 n+1 \\
k
\end{array}\right] \varphi\left(q^{-k} ; n\right) \varphi\left(\lambda q^{k} ; n\right) \psi\left(q^{-k} ; n+1\right) \psi\left(\lambda q^{k} ; n+1\right) \\
& \times \frac{1-\lambda q^{2 k}}{\left(\lambda q^{2 n+1} ; q\right)_{k+1}} \Omega(k),
\end{aligned}
$$

provided that the sequence-transforms involved are non-singular, i.e. $\varphi\left(\lambda q^{n} ; m\right), \varphi\left(q^{-n} ; m\right), \varphi\left(\lambda q^{n} ; m+1\right), \varphi\left(q^{-n} ; m+1\right), \psi\left(\lambda q^{n} ; m+1\right)$ $\psi\left(q^{-n} ; m+1\right),\left(\lambda q^{2 n} ; m+1\right)$ and $\left(\lambda q^{2 n+1} ; m+1\right)$ do not vanish for non-negative integers $m \leq n$.

\section{Applications}

Using Theorem 2.2, we derive several terminating basic hypergeometric identities and some limiting cases.

Theorem 3.1.

$$
\begin{aligned}
& \sum_{k=0}^{n}\left[\begin{array}{c}
q^{-n}, a q^{n}, \frac{a}{b}, b \\
q, \frac{b q^{1-n}}{a}, b q^{1+n}, \frac{b^{2} q}{a} ; q
\end{array}\right]_{k}\left[\begin{array}{c}
\frac{b^{2} q}{a}, b q^{2} ; q^{2} \\
a q, b
\end{array}\right]_{k}\left(-\frac{b q}{a}\right)^{k} \\
& =\left\{\begin{array}{l}
{\left[\begin{array}{l}
b q, b q^{2}, \frac{a^{2}}{b^{2}}, q \\
a q, \frac{a}{b}, \frac{a q}{b}, \frac{b^{2} q^{2}}{a} ; q^{2}
\end{array}\right]_{m}, \quad n=2 m ;} \\
0, \quad n=2 m+1 .
\end{array}\right.
\end{aligned}
$$


Proof. By the replacement $q \rightarrow q^{2}$, (1.5) can be rewritten as

$$
\begin{aligned}
& { }_{8} \phi_{7}\left[\begin{array}{l}
a, q^{2} a^{\frac{1}{2}},-q^{2} a^{\frac{1}{2}}, \frac{a^{2}}{b^{2}}, b q^{n}, b q^{1+n}, q^{-n}, q^{-(n-1)} ; q^{2}, q^{2} \\
a^{\frac{1}{2}},-a^{\frac{1}{2}}, \frac{b^{2} q^{2}}{a}, \frac{a q^{2-n}}{b}, \frac{a q^{1-n}}{b}, a q^{2+n}, a q^{1+n}
\end{array}\right] \\
& \left.=\left(-\frac{b}{a}\right)^{n}\left[\begin{array}{l}
a q, \frac{a}{b} \\
\frac{b^{2} q}{a}, \frac{b}{a}
\end{array}\right]_{n}\right]\left[\begin{array}{l}
\frac{b^{2} q}{a} ; q^{2} \\
a q
\end{array}\right]_{n} .
\end{aligned}
$$

Assume

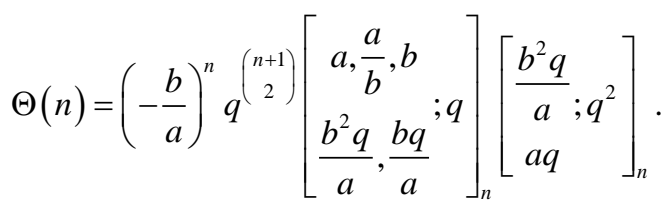

Let us multiply both sides of (3.1) by $\frac{q^{\left(\begin{array}{l}n \\ 2\end{array}\right)\left(1-\frac{a}{b}\right)(b ; q)_{n}(1-a)}}{\left(1-\frac{a q^{-n}}{b}\right)\left(1-a q^{n}\right)}$. Since

$$
\begin{aligned}
& \frac{\left(q^{-n}, q^{-(n-1)} ; q^{2}\right)_{k}\left(q ; q^{2}\right)_{k}}{\left(q^{2}, q ; q^{2}\right)_{k}}=\frac{\left(q^{-n} ; q\right)}{(q ;} \\
& q^{\left(\begin{array}{c}
n \\
2
\end{array}\right)} \frac{\left(q^{-n} ; q\right)_{2 k}}{(q ; q)_{2 k}} q^{2 k}=\left[\begin{array}{c}
n \\
2 k
\end{array}\right] q^{\left(\begin{array}{c}
n-2 k \\
2
\end{array}\right),}
\end{aligned}
$$

we have

$$
\begin{aligned}
\Theta(n)= & \sum_{k \geq 0}\left[\begin{array}{c}
n \\
2 k
\end{array}\right] q^{\left(\begin{array}{c}
n-2 k \\
2
\end{array}\right)} \frac{\left(1-a q^{4 k}\right)\left(1-\frac{a}{b}\right)\left(b q^{2 k} ; q\right)_{n}}{\left(\frac{a q^{1-n}}{b} ; q^{2}\right)_{k}\left(a q^{1+n} ; q^{2}\right)_{k}\left(\frac{a q^{-n}}{b} ; q^{2}\right)_{k+1}\left(a q^{n} ; q^{2}\right)_{k+1}} \\
& \times \frac{(b ; q)_{2 k}\left(a, \frac{a^{2}}{b^{2}}, q ; q^{2}\right)_{k}}{\left(\frac{b^{2} q^{2}}{a} ; q^{2}\right)_{k}} .
\end{aligned}
$$

It is not difficult to see that (2.9) specified with

$$
\lambda=b, \quad \varphi(x ; n)=\left(\frac{a q x}{b} ; q^{2}\right)_{n} \text { and } \psi(x ; n)=\left(\frac{a x}{b} ; q^{2}\right)_{n}
$$

matches exactly (3.2) under the following settings

$$
\Omega(n)=\Theta(n), \quad f(n)=\frac{(b ; q)_{2 n}\left(a, \frac{a^{2}}{b^{2}}, q ; q^{2}\right)_{n}}{\left(\frac{b^{2} q^{2}}{a} ; q^{2}\right)_{n}} \text { and } g(n)=0 .
$$


The dual relations corresponding to (2.10) and (2.11) result respectively in the following two formulae

$$
\begin{aligned}
& \sum_{k=0}^{2 n}(-1)^{k}\left[\begin{array}{c}
2 n \\
k
\end{array}\right]\left(\frac{a q^{1-k}}{b} ; q^{2}\right)_{n}\left(a q^{1+k} ; q^{2}\right)_{n}\left(\frac{a q^{-k}}{b} ; q^{2}\right)_{n}\left(a q^{k} ; q^{2}\right)_{n} \\
& \times \frac{1-b q^{2 k}}{\left(b q^{2 n} ; q\right)_{k+1}} \Omega(k)=f(n), \\
& \sum_{k=0}^{2 n+1}(-1)^{k}\left[\begin{array}{c}
2 n+1 \\
k
\end{array}\right]\left(\frac{a q^{1-k}}{b} ; q^{2}\right)_{n}\left(a q^{1+k} ; q^{2}\right)_{n}\left(\frac{a q^{-k}}{b} ; q^{2}\right)_{n+1}\left(a q^{k} ; q^{2}\right)_{n+1} \\
& \times \frac{1-b q^{2 k}}{\left(b q^{2 n+1} ; q\right)_{k+1}} \Omega(k)=0 .
\end{aligned}
$$

Notice that

$$
\begin{aligned}
& (-1)^{k}\left[\begin{array}{c}
2 n \\
k
\end{array}\right]=\frac{q^{2 n k-\left(\begin{array}{l}
k \\
2
\end{array}\right)\left(q^{-2 n} ; q\right)_{k}}}{(q ; q)_{k}}, \quad(-1)^{k}\left[\begin{array}{c}
2 n+1 \\
k
\end{array}\right]=\frac{q^{(2 n+1) k-\left(\begin{array}{c}
k \\
2
\end{array}\right)}\left(q^{-(2 n+1)} ; q\right)_{k}}{(q ; q)_{k}}, \\
& \left(a q^{1+k} ; q^{2}\right)_{n}\left(a q^{k} ; q^{2}\right)_{n}=\left(a q^{k} ; q\right)_{2 n}=\frac{(a ; q)_{2 n}\left(a q^{2 n} ; q\right)_{k}}{(a ; q)_{k}} \text {, } \\
& \left(\frac{a q^{1-k}}{b} ; q^{2}\right)_{n}\left(\frac{a q^{-k}}{b} ; q^{2}\right)_{n}=\left(\frac{a q^{-k}}{b} ; q\right)_{2 n}=\frac{\left(\frac{a q^{-k}}{b} ; q\right)_{k}\left(\frac{a}{b} ; q\right)_{2 n}}{\left(\frac{a q^{2 n-k}}{b} ; q\right)_{k}}=\frac{q^{-2 n k}\left(\frac{b q}{a} ; q\right)_{k}\left(\frac{a}{b} ; q\right)_{2 n}}{\left(\frac{b q^{1-2 n}}{a} ; q\right)_{k}} .
\end{aligned}
$$

After some simplification, we obtain Theorem 3.1.

Remark 1. Theorem 3.1 is not only a result which Chu obtained in [5], it is actually already a special case of an identity due to Jain and Verma, listed in [1] as (2.16) (where $a, b, c$, and $\lambda$, are specialized to $a q^{n}, q^{-n},-b \sqrt{\frac{q}{a}}$, and $b$, respectively).

Theorem 3.2.

$$
\begin{aligned}
& \sum_{k=0}^{n}\left[\begin{array}{c}
q^{-n}, a q^{n-1}, \frac{a}{b}, b \\
\left.q, \frac{b q^{2-n}}{a}, b q^{1+n}, \frac{b^{2} q}{a} ; q\right]_{k}\left[\begin{array}{c}
\frac{b^{2} q}{a}, b q^{2} ; q^{2} \\
a q, b
\end{array}\right]_{k}\left(-\frac{b q^{2}}{a}\right)^{k} \\
\frac{(b q ; q)_{2 m}\left(a, \frac{a^{2}}{b^{2}}, q ; q^{2}\right)_{m}}{\left(1-a q^{4 m-1}\right)\left(\frac{b^{2} q^{2}}{a} ; q^{2}\right)\left(\frac{a}{b q} ; q\right)_{2 m}(a ; q)_{2 m-1}}, \quad n=2 m ; \\
\frac{(b q ; q)_{2 m+1}\left(a, \frac{a^{2}}{b^{2}}, q ; q^{2}\right)\left(1-q^{2 m+1}\right)\left(\frac{-a}{b q}\right)}{\left(1-a q^{4 m+1}\right)\left(\frac{b^{2} q^{2}}{a} ; q^{2}\right)_{m}\left(\frac{a}{b q} ; q\right)_{2 m+1}(a ; q)_{2 m}}, \quad n=2 m+1 .
\end{array}\right.
\end{aligned}
$$


Proof Multiplying both sides of (3.2) by $\frac{1}{\left(1-\frac{a q^{-n-1}}{b}\right)\left(1-a q^{n-1}\right)}$, then applying the relation

$$
\left(1-a q^{4 k}\right)\left(1-\frac{a}{b}\right)=\left(1-\frac{a q^{-n+2 k}}{b}\right)\left(1-a q^{n+2 k}\right)-q^{1+2 k-n}\left(1-b q^{2 k+n}\right)\left(1-q^{n-2 k}\right)\left(\frac{-a}{b q}\right),
$$

we may restate the resulting identity as

$$
\begin{aligned}
& \frac{\left(-\frac{b}{a}\right)^{n} q^{\left(\begin{array}{c}
n+1 \\
2
\end{array}\right)}}{\left(1-\frac{a q^{-n-1}}{b}\right)\left(1-a q^{n-1}\right)}\left[\begin{array}{c}
a, \frac{a}{b}, b \\
\frac{b^{2} q}{a}, \frac{b q}{a} ; q
\end{array}\right]_{n}\left[\begin{array}{c}
\frac{b^{2} q}{a} ; q^{2} \\
a q
\end{array}\right]_{n} \\
& =\sum_{k \geq 0}\left[\begin{array}{c}
n \\
2 k
\end{array}\right] q^{\left(\begin{array}{c}
n-2 k \\
2
\end{array}\right)} \frac{\left(1-a q^{4 k-1}\right)\left(1-\frac{a}{b q}\right)\left(b q^{2 k} ; q\right)_{n}}{\left(\frac{a q^{-n}}{b} ; q^{2}\right)_{k}\left(a q^{n} ; q^{2}\right)_{k}\left(\frac{a q^{-n-1}}{b} ; q^{2}\right)_{k+1}\left(a q^{n-1} ; q^{2}\right)_{k+1}} \\
& \times \frac{(b ; q)_{2 k}\left(a, \frac{a^{2}}{b^{2}}, q ; q^{2}\right)_{k}}{\left(1-a q^{4 k-1}\right)\left(1-\frac{a}{b q}\right)\left(\frac{b^{2} q^{2}}{a} ; q^{2}\right)_{k}} \\
& -\sum_{k \geq 0}\left[\begin{array}{c}
n \\
2 k+1
\end{array}\right] q^{\left(\begin{array}{c}
n-2 k-1 \\
2
\end{array}\right)} \frac{\left(1-a q^{4 k+1}\right)\left(1-\frac{a}{b q}\right)\left(b q^{2 k+1} ; q\right)_{n}}{\left(\frac{a q^{-n}}{b} ; q^{2}\right)_{k+1}\left(a q^{n} ; q^{2}\right)_{k+1}\left(\frac{a q^{-n-1}}{b} ; q^{2}\right)_{k+1}\left(a q^{n-1} ; q^{2}\right)_{k+1}} \\
& \times \frac{(b ; q)_{2 k+1}\left(a, \frac{a^{2}}{b^{2}}, q ; q^{2}\right)_{k}\left(1-q^{2 k+1}\right)\left(\frac{-a}{b q}\right)}{\left(1-a q^{4 k-1}\right)\left(1-\frac{a}{b q}\right)\left(\frac{b^{2} q^{2}}{a} ; q^{2}\right)_{k}} .
\end{aligned}
$$

It is not difficult to see that (2.9) specified with

$$
\lambda=b, \quad \varphi(x ; n)=\left(\frac{a x}{b} ; q^{2}\right)_{n} \text { and } \psi(x ; n)=\left(\frac{a q^{-1} x}{b} ; q^{2}\right)_{n}
$$

matches exactly (3.3) under the following settings

$$
\begin{gathered}
\Omega(n)=\frac{\left(-\frac{b}{a}\right)^{n} q^{\left(\begin{array}{c}
n+1 \\
2
\end{array}\right)}}{\left(1-\frac{a q^{-n-1}}{b}\right)\left(1-a q^{n-1}\right)}\left[\begin{array}{c}
a, \frac{a}{b}, b \\
\frac{b^{2} q}{a}, \frac{b q}{a} ; q
\end{array}\right]_{n}\left[\begin{array}{c}
\frac{b^{2} q}{a} ; q^{2} \\
a q
\end{array}\right]_{n}, \\
f(n)=\frac{(b ; q)_{2 n}\left(a, \frac{a^{2}}{b^{2}}, q ; q^{2}\right)_{n}}{\left(1-a q^{4 n-1}\right)\left(1-\frac{a}{b q}\right)\left(\frac{b^{2} q^{2}}{a} ; q^{2}\right)_{n}},
\end{gathered}
$$




$$
g(n)=\frac{(b ; q)_{2 n+1}\left(a, \frac{a^{2}}{b^{2}}, q ; q^{2}\right)_{n}\left(1-q^{2 n+1}\right)\left(\frac{-a}{b q}\right)}{\left(1-a q^{4 n+1}\right)\left(1-\frac{a}{b q}\right)\left(\frac{b^{2} q^{2}}{a} ; q^{2}\right)_{n}} .
$$

The dual relations corresponding to (2.10) and (2.11) result respectively in the following two formulae

$$
\begin{aligned}
& \sum_{k=0}^{2 n}(-1)^{k}\left[\begin{array}{c}
2 n \\
k
\end{array}\right]\left(\frac{a q^{-k}}{b} ; q^{2}\right)_{n}\left(a q^{k} ; q^{2}\right)_{n}\left(\frac{a q^{-1-k}}{b} ; q^{2}\right)_{n}\left(a q^{-1+k} ; q^{2}\right)_{n} \\
& \times \frac{1-b q^{2 k}}{\left(b q^{2 n} ; q\right)_{k+1}} \Omega(k)=f(n), \\
& \sum_{k=0}^{2 n+1}(-1)^{k}\left[\begin{array}{c}
2 n+1 \\
k
\end{array}\right]\left(\frac{a q^{-k}}{b} ; q^{2}\right)_{n}\left(a q^{k} ; q^{2}\right)_{n}\left(\frac{a q^{-1-k}}{b} ; q^{2}\right)_{n+1}\left(a q^{-1+k} ; q^{2}\right)_{n+1} \\
& \times \frac{1-b q^{2 k}}{\left(b q^{2 n+1} ; q\right)_{k+1}} \Omega(k)=g(n) .
\end{aligned}
$$

Carrying out the straightforward calculations, we get the desired result.

Theorem 3.3.

$$
\begin{aligned}
& \sum_{k=0}^{2 n+1}\left[\begin{array}{c}
q^{-2 n-1}, \frac{b q^{1-2 n}}{a}, a q^{2 n+1}, a q^{2 n-1}, \frac{a}{b}, b \\
q, \frac{b q^{2-2 n}}{a}, \frac{b q^{-2 n}}{a}, b q^{2 n+2}, a q^{2 n}, \frac{b^{2} q}{a} ; q
\end{array}\right]_{k}\left[\begin{array}{c}
\frac{b^{2} q}{a}, b q^{2} ; q^{2} \\
a q, b
\end{array}\right]_{k}\left(-\frac{b q^{2}}{a}\right)^{k} \\
& =\frac{\left(1-q^{2 n+1}\right)\left(1-a q^{2 n-1}\right)\left(1-\frac{a q^{2 n-1}}{b}\right)(b q ; q)_{2 n+1}\left(a, \frac{a^{2}}{b^{2}}, q ; q^{2}\right)_{n}}{\left(1-a q^{4 n-1}\right)\left(1-\frac{a}{b q}\right)\left(1-\frac{b q^{2}}{a}\right)\left(\frac{a q}{b} ; q\right)_{2 n}(a ; q)_{2 n+1}\left(\frac{b^{2} q^{2}}{a} ; q^{2}\right)_{n}} .
\end{aligned}
$$

Proof. (3.2) can be rewritten as follows

$$
\begin{aligned}
& \left(-\frac{b}{a}\right)^{n} q^{\left(\begin{array}{c}
n+1 \\
2
\end{array}\right)}\left[\begin{array}{c}
a, \frac{a}{b}, b \\
\frac{b^{2} q}{a}, \frac{b q}{a} ; q
\end{array}\right]_{n}\left[\begin{array}{c}
\frac{b^{2} q}{a} ; q^{2} \\
a q
\end{array}\right]_{n} \\
& =\sum_{k \geq 0}\left[\begin{array}{c}
n \\
2 k
\end{array}\right] q^{\left(\begin{array}{c}
n-2 k \\
2
\end{array}\right)} \frac{\left(1-a q^{4 k}\right)\left(1-\frac{a}{b}\right)\left(1-a q^{4 k-1}\right)\left(1-\frac{a}{b q}\right)\left(b q^{2 k} ; q\right)_{n}}{\left(\frac{a q^{1-n}}{b} ; q^{2}\right)_{k}\left(a q^{1+n} ; q^{2}\right)_{k}\left(\frac{a q^{-n}}{b} ; q^{2}\right)_{k+1}\left(a q^{n} ; q^{2}\right)_{k+1}} \\
& \times \frac{(b ; q)_{2 k}\left(a, \frac{a^{2}}{b^{2}}, q ; q^{2}\right)_{k}}{\left(1-a q^{4 k-1}\right)\left(1-\frac{a}{b q}\right)\left(\frac{b^{2} q^{2}}{a} ; q^{2}\right)_{k}} .
\end{aligned}
$$

Multiplying both sides of (3.5) by $\frac{1}{\left(1-\frac{a q^{-n-1}}{b}\right)\left(1-a q^{n-1}\right)}$, then applying the 
relation

$$
\begin{aligned}
& \left(1-a q^{4 k-1}\right)\left(1-\frac{a}{b q}\right) \\
& =\left(1-\frac{a q^{-n-1+2 k}}{b}\right)\left(1-a q^{n+2 k-1}\right)-q^{1+2 k-n}\left(1-b q^{2 k+n}\right)\left(1-q^{n-2 k}\right)\left(\frac{-a}{b q^{2}}\right),
\end{aligned}
$$

we have

$$
\begin{aligned}
& \frac{\left(-\frac{b}{a}\right)^{n} q^{\left(\begin{array}{c}
n+1 \\
2
\end{array}\right)}}{\left(1-\frac{a q^{-n-1}}{b}\right)\left(1-a q^{n-1}\right)}\left[\frac{b^{2} q}{a}, \frac{a}{b}, \frac{b q}{a} ; q\right]_{n}\left[\begin{array}{c}
\frac{b^{2} q}{a} ; q^{2} \\
a q
\end{array}\right]_{n} \\
& =\sum_{k \geq 0}\left[\begin{array}{c}
n \\
2 k
\end{array}\right] q^{\left(\begin{array}{c}
n-2 k \\
2
\end{array}\right)} \frac{\left(1-a q^{4 k}\right)\left(1-\frac{a}{b}\right)\left(b q^{2 k} ; q\right)_{n}}{\left(\frac{a q^{-n-1}}{b} ; q^{2}\right)_{k}\left(a q^{n-1} ; q^{2}\right)_{k}\left(\frac{a q^{-n}}{b} ; q^{2}\right)_{k+1}\left(a q^{n} ; q^{2}\right)_{k+1}} \\
& \times \frac{(b ; q)_{2 k}\left(a, \frac{a^{2}}{b^{2}}, q ; q^{2}\right)_{k}}{\left(1-a q^{4 k-1}\right)\left(1-\frac{a}{b q}\right)\left(\frac{b^{2} q^{2}}{a} ; q^{2}\right)_{k}} \\
& -\sum_{k \geq 0}\left[\begin{array}{c}
n \\
2 k+1
\end{array}\right] q^{\left(\begin{array}{c}
n-2 k-1 \\
2
\end{array}\right)} \frac{\left(1-a q^{4 k}\right)\left(1-\frac{a}{b q^{2}}\right)\left(b q^{2 k+1} ; q\right)_{n}}{\left(\frac{a q^{-n-1}}{b} ; q^{2}\right)_{k+1}\left(a q^{n-1} ; q^{2}\right)_{k+1}\left(\frac{a q^{-n}}{b} ; q^{2}\right)_{k+1}\left(a q^{n} ; q^{2}\right)_{k+1}} \\
& \times \frac{(b ; q)_{2 k}\left(a, \frac{a^{2}}{b^{2}}, q ; q^{2}\right)_{k}\left(1-q^{2 k+1}\right)\left(1-b q^{2 k}\right)\left(1-\frac{a}{b}\right)\left(\frac{-a}{b q^{2}}\right)}{\left(1-a q^{4 k-1}\right)\left(1-\frac{a}{b q}\right)\left(1-\frac{a}{b q^{2}}\right)\left(\frac{b^{2} q^{2}}{a} ; q^{2}\right)_{k}} .
\end{aligned}
$$

It is easy to see that (2.9) specified with

$$
\lambda=b, \quad \varphi(x ; n)=\left(\frac{a q^{-1} x}{b} ; q^{2}\right)_{n} \text { and } \quad \psi(x ; n)=\left(\frac{a x}{b} ; q^{2}\right)_{n}
$$

matches exactly (3.6) under the following settings

$$
\begin{gathered}
\Omega(n)=\frac{\left(-\frac{b}{a}\right)^{n} q^{\left(\begin{array}{c}
n+1 \\
2
\end{array}\right)}}{\left(1-\frac{a q^{-n-1}}{b}\right)\left(1-a q^{n-1}\right)}\left[\begin{array}{c}
a, \frac{a}{b}, b \\
\frac{b^{2} q}{a}, \frac{b q}{a} ; q
\end{array}\right]_{n}\left[\begin{array}{c}
\frac{b^{2} q}{a} ; q^{2} \\
a q
\end{array}\right]_{n}, \\
f(n)=\frac{(b ; q)_{2 n}\left(a, \frac{a^{2}}{b^{2}}, q ; q^{2}\right)_{n}}{\left(1-a q^{4 n-1}\right)\left(1-\frac{a}{b q}\right)\left(\frac{b^{2} q^{2}}{a} ; q^{2}\right)_{n}},
\end{gathered}
$$




$$
g(n)=\frac{\left(1-q^{2 n+1}\right)\left(1-\frac{a}{b}\right)\left(\frac{-a}{b q^{2}}\right)(b ; q)_{2 n+1}\left(a, \frac{a^{2}}{b^{2}}, q ; q^{2}\right)_{n}}{\left(1-a q^{4 n-1}\right)\left(1-\frac{a}{b q}\right)\left(1-\frac{a}{b q^{2}}\right)\left(\frac{b^{2} q^{2}}{a} ; q^{2}\right)_{n}} .
$$

The dual relation corresponding to (2.10) is (3.4), and the dual relation corresponding to (2.11) is

$$
\begin{aligned}
& \sum_{k=0}^{2 n+1}(-1)^{k}\left[\begin{array}{c}
2 n+1 \\
k
\end{array}\right]\left(\frac{a q^{-k-1}}{b} ; q^{2}\right)_{n}\left(a q^{k-1} ; q^{2}\right)_{n}\left(\frac{a q^{-k}}{b} ; q^{2}\right)_{n+1}\left(a q^{k} ; q^{2}\right)_{n+1} \\
& \times \frac{1-b q^{2 k}}{\left(b q^{2 n+1} ; q\right)_{k+1}} \Omega(k)=g(n) .
\end{aligned}
$$

Carrying out the straightforward calculations, we get the desired result.

Taking some special limits, we have the following cases.

Case $1 \quad b \rightarrow \infty$ in Theorem 3.2:

$$
\begin{aligned}
& \sum_{k=0}^{n}\left[\begin{array}{c}
q^{-n}, a q^{n-1} ; q \\
q
\end{array}\right]_{k} \frac{q^{k}}{\left(a q ; q^{2}\right)_{k}} \\
& = \begin{cases}\frac{\left(a, q ; q^{2}\right)_{m}(-1)^{m} a^{m} q^{m^{2}}}{\left(1-a q^{4 m-1}\right)(a ; q)_{2 m-1}}, & n=2 m ; \\
\frac{\left(a, q ; q^{2}\right)_{m}\left(1-q^{2 m+1}\right)(-1)^{m} a^{m+1} q^{m^{2}+2 m}}{\left(1-a q^{4 m+1}\right)(a ; q)_{2 m}}, & n=2 m+1 .\end{cases}
\end{aligned}
$$

Case $2 b \rightarrow \infty, a \rightarrow \infty$ in Theorem 3.2:

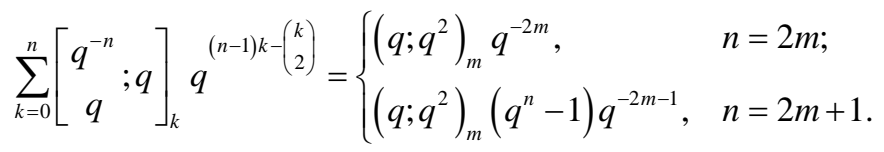

Case $3 a=b q^{2}$ in Theorem 3.2:

$$
\sum_{k=0}^{n} \frac{\left(1-q^{k+1}\right)\left(1-b q^{k-1}\right)\left(1-b q^{2 k}\right)(-1)^{k}}{\left(1-b q^{1+2 k}\right)\left(1-b q^{2 k-1}\right)}= \begin{cases}\frac{\left(1-b q^{2 m}\right)\left(1-q^{2 m+2}\right)}{\left(1-b q^{4 m+1}\right)(1+q)}, & n=2 m ; \\ \frac{\left(1-b q^{2 m}\right)\left(1-q^{2 m+2}\right)(-q)}{\left(1-b q^{4 m+3}\right)(1+q)}, & n=2 m+1 .\end{cases}
$$

Case $4 \quad b \rightarrow 0$ in Theorem 3.2:

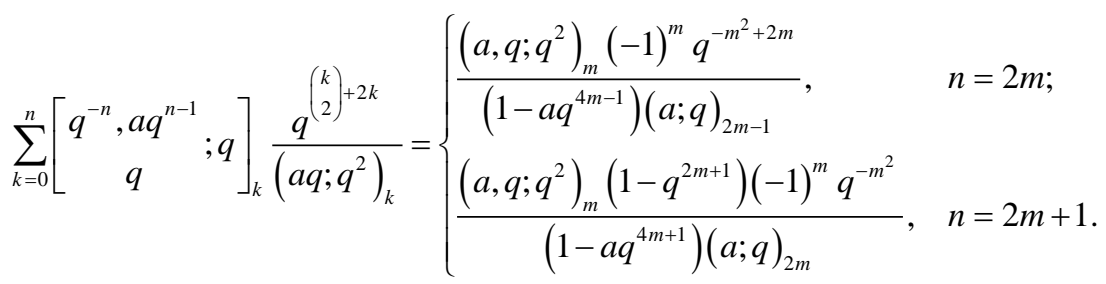

Case $5 b \rightarrow 0, a=0$ in Theorem 3.2:

$$
\sum_{k=0}^{n}\left[\begin{array}{c}
q^{-n} ; q \\
q
\end{array}\right]_{k} q^{\left(\begin{array}{c}
k \\
2
\end{array}\right)+2 k}= \begin{cases}\left(q ; q^{2}\right)_{m}(-1)^{m} q^{-m^{2}+2 m}, & n=2 m ; \\
\left(q ; q^{2}\right)_{m}\left(1-q^{2 m+1}\right)(-1)^{m} q^{-m^{2}}, & n=2 m+1 .\end{cases}
$$




\section{Conflicts of Interest}

The author declares no conflicts of interest regarding the publication of this paper.

\section{References}

[1] Gasper, G. and Rahman, M. (2004) Basic Hypergeometric Series. 2nd Edition, Cambridge University Press, Cambridge. https://doi.org/10.1017/CBO9780511526251

[2] Gould, H.W. and Hsu, L.C. (1973) Some New Inverse Series Relations. Duke Mathematical Journal, 40, 885-891. https://doi.org/10.1215/S0012-7094-73-04082-9

[3] Carlitz, L. (1973) Some Inverse Relations. Duke Mathematical Journal, 40, 893-901. https://doi.org/10.1215/S0012-7094-73-04083-0

[4] Chu, W.C. (1994) Inversion Techniques and Combinatorial Identities: A Unified Treatment for the ${ }_{7} F_{6}$-Series Identities. Collectanea Mathematica, 45, 13-43.

[5] Chu, W.C (1995) Inversion Techniques and Combinatorial Identities: Jackson's $q$-Analogue of the Dougall-Dixon Theorem and the Dual Foemulae. Compositio Mathematica, 95, 43-68.

[6] Chu, W.C. (2002) Inversion Techniques and Combinatorial Identities: Balanced Hypergeometric Series. Rocky Mountain Journal of Mathematics, 32, 561-587. https://doi.org/10.1216/rmjm/1030539687

[7] Wei, C.N., Gong, D.X. and Fu, X.M. (2011) Duplicate form of Carlitz Inversions and Summation. Discrete Mathematics, 311, 1699-1713. https://doi.org/10.1016/j.disc.2011.04.011

[8] Chen, X.J. and Chu, W.C. (2011) The Pfaff-Saalschütz Theorem and Terminating ${ }_{3} F_{2}\left(\frac{4}{3}\right)$ Series. Journal of Mathematical Analysis and Applications, 380, 440-454.

[9] Wei, C.N., Gong, D.X. and Hao, H.Y. (2012) A Family of Summation Formulas on the Fox-Wright Function. Journal of Mathematical Analysis and Applications, 393, 316-327. https://doi.org/10.1016/j.jmaa.2012.03.008

[10] Shi, J. and Zhang, W.L. (2017) Summation Formulae on Terminating ${ }_{6} F_{5}(-1)$ Series. Journal of Mathematical Analysis and Applications, 456, 1005-1012. https://doi.org/10.1016/j.jmaa.2017.07.034

[11] Zhang, Z.Z. and Li, N. (2011) Some $q$-Series Identity Related to the $q$-Triplicate Inverse. Rocky Mountain Journal of Mathematics, 41, 1375-1393. https://doi.org/10.1216/RMJ-2011-41-4-1375 